\title{
4-Hydroxy-2-Nonenal, Indused Nitric Oxide Synthase Statues in Hypertension Patients
}

\author{
Ali D. Hammed ${ }^{1}$, Alaa H. Jawad ${ }^{1 *}$ and Perry H. Saifullah ${ }^{2}$ \\ ${ }^{1}$ Department of Chemistry, College of Science, Al-Nahrain University, Baghdad-Iraq. \\ ${ }^{2}$ Department of Chemistry, College of Science for women, Baghdad University, Baghdad-Iraq. \\ *Corresponding Author: Alaaalqaisi74@yahoo.com.
}

\begin{abstract}
The oxidative stress is one of the main cause for cardiovascular diseases (like Hypertension) also one of the results of these diseases. This study involved 56 subjects matched ages and sex divided into two groups; 28 hypertensive subject and 28 healthy subject as control group. The following analysis was done: 4-Hydroxy-2-nonenal (4HNE), Induced nitric oxide synthase (iNOS) and albumin. The results show that there is a significant increase in (4HNE) between patients group and control group. INOS was significantly higher in patients compared to controls while there were no significant difference found in albumin between patient and control group. The increase in $4 \mathrm{HNE}$ which is a product of lipid peroxidation is attributed to destruction in body cell caused by due to the increase in stress events. And the increase in iNOS because iNOS produces large amounts of NO as a defense mechanism in response to cytokines and is an important factor in the response of the body to attack by parasites, bacterial infection, and tumor growth. It has been concluded that is important nappy on ideal weight, because obesity considered main factors for heart disease and hardening of the arteries. There is a positive relationship between oxidation results from hypertension and their developments. The aim of this study was to investigate the relationship between of some oxidative stress markers and cardiovascular diseases. [DOI: 10.22401/JNUS.20.3.02]
\end{abstract}

Keywords: Hypertension, 4-Hydroxy-2-nonenal (4HNE), Induced nitric oxide synthase (iNOS), Reactive oxygen species (ROS).

\section{Introduction}

Blood pressure is a measure of the force exercised circulation on the walls of the major arteries. hypertension is a chronic medical case which in the blood pressure in the arteries is elevated[1]. Macrophages had been supposed to be the source of the most reactive oxygen species in the vessel's wall, However, it has become clear that all the cells in the vessel wall produce ROS in different quantities and in response to diverse stimuli [2]. Cardiovascular disease is a pathological condition interrelated with cardiac valves, Oxidative stress promotes vascular smooth muscle cell proliferation and hypertrophy and collagen deposition, which leading to thickening in the vascular media and narrowing of the vascular cavity [3]. It has been shown that mechanical stretch to vessel wall induces ROS release. This suggests the possibility that high blood pressure itself causes raises to ROS independent of renin angiotensin system activity [3]. In addition, increased oxidative stress causes tissue damage by different mechanisms including promoting lipid peroxidation, DNA damage, and protein modification. [4]. Reactive oxygen species (ROS) are highly reactive intermediates of the oxygen metabolism, which are constantly being generated and destroyed. ROS may originate from both exogenous and endogenous sources [5]. Exogenous sources include environmental agents (like, UV or heat exposure), ionizing radiation, therapeutic agents, and tobacco smoke. Endogenous sources include mitochondria, peroxisome and inflammatory cell activation [5]. When there is an imbalance between the generation of ROS and the antioxidant defense system so that the latter becomes overwhelmed, oxidative stress occurs [2]. Oxidants and free radicals are inevitably produced during most physiological and metabolical processes, and the human body has defensive antioxidant mechanisms, these mechanisms vary according to the cell and tissue type and they may act antagonistically or synergistically [6]. Oxidative stress leads to many pathophysiological conditions in the body [7]. including neurodegenerative diseases like Parkinson's disease and Alzheimer's disease, gene mutations and cancers, chronic 
fatigue syndrome, heart and blood vessel Disturbance, atherosclerosis, heart, congestive heart attack and inflammatory diseases[8]. In physiological conditions, low level ROS play a role in the protection the organism, while high levels of ROS may cause damage to the structures of the cell, nucleic acids, lipids, proteins or DNA damage [9]. 4-hydroxy-2nonenal (4HNE), a high toxicity product of lipid peroxidation, is an inhibitor of mitochondrial respiration. 4HNE It exerts its influence on respiration by inhibiting $\alpha$-ketoglutarate dehydrogenase (KGDH) [10]. a study by Teresa S.et al [11] recorded a significant increase in $4 \mathrm{HNE}$ values in hypertension patients and Juliane Cruz Campos et al 2015 observed an increase in 4HNE with hypertension and kidney disease [12]. Nitric oxide synthases (NOSs) are a family of enzymes catalyzing the production of nitric oxide (NO) from L-arginine [13]. NO is an important cellular signaling molecule. Albumin represents the most abundant protein in the circulatory system with a significant antioxidant activity [14], the antioxidant activity of albumin result from its ability to bind bilirubin, homocysteine and lipids [15] The results of the present study showed that the levels of albumin was significantly decrease $(\mathrm{p}<0.01)$ in diagnosed hypertension and hypertension with kidney patients which is agree with (Oda E) in hypertension.[15]

\section{Materials and Methods}

The present study comprised of 56 men divide to two groups [namely control group (28), hypertensive group (28)] aged between 22-65 years. These patients were hospitalized at educational laboratories in the Al-Yarmouk teaching hospital. They were divided into groups of healthy as control group and hypertensive patients group. Blood sample were collected and centrifuged at $[4000 \mathrm{xg}$ ] for 5 min after clotting. The resultant serum were separated and stored at $[-20]^{\circ} \mathrm{C}$ until used. Estimation of serum albumin was done using kit provided by Bio Systems company.

Serum 4-Hydroxy-2-nonenal 4HNE is typically quantified from serum samples with the most popular method being a colorimetric assay based on biotin double antibody sandwich technology.
Serum Indused nitric oxide synthase iNOS is typically quantified from serum samples the assay employs the quantitative sandwich enzyme immunoassay technique.

\section{Statistics}

The Statistical Analysis System-SAS (2012) was used to determine of different factors in studied parameters, P-value used to significant compare between means in this study.

\section{Results and Discussion}

Fifty six sample subjects comprising of 28 patients and 28 healthy were included in the present study, Table (1) shows the means and standard deviation of age, body mass index (BMI), duration of disease, 4-Hydroxy-2nonenal (4HNE) in addition to albumin levels for the control and patients groups. 
Table (1)

Characteristics of the Hypertension (HT) and control group (mean $\pm S D)$.

\begin{tabular}{|c||c||c||c||}
\hline Characteristic & $\begin{array}{c}\text { Hypertension } \\
\text { group } \\
\mathbf{n = 2 8}\end{array}$ & $\begin{array}{c}\text { Control group } \\
\mathbf{n = 2 8}\end{array}$ & P-Value \\
\hline \hline Age (year) & $55.46 \pm 8.91$ & $23.82 \pm 5.02$ & $<0.01$ \\
\hline \hline Body Mass index $\left(\mathrm{Kg} / \mathrm{m}^{2}\right)$ & $33.34 \pm 4.68$ & $25.18 \pm 2.89$ & $<0.01$ \\
\hline Systolic blood pressure $(\mathrm{mmHg})$ & $14.60 \pm 1.22$ & $11.96 \pm 0.66$ & $<0.01$ \\
\hline Diastolic blood pressure $(\mathrm{mmHg})$ & $9.23 \pm 0.85$ & $8.63 \pm 0.56$ & $<0.01$ \\
\hline 4HNE (ng/L) & $160.22 \pm 42.63$ & $136.46 \pm 24.55$ & $<0.01$ \\
\hline iNOS (IU/ml) & $10.11 \pm 7.08$ & $6.42 \pm 4.93$ & $<0.01$ \\
\hline Albumin (g/dl) & $4.38 \pm 0.27$ & $4.73 \pm 0.25$ & $<0.01$ \\
\hline
\end{tabular}

There is a significant differences $(\mathrm{p}<0.01)$ in age when comparing patients groups with control group. The significant difference in BMI $(\mathrm{p}<0.01)$ between HT and control group reveal the positive correlation between obesity and disease. This finding was similar to (Ahmed A. et al 2013) and (Shugar L et al 2008) they found a significant increase in BMI in hypertensive patients [16,17]. Obese patients are more able to be hypertensive than lean patients, and weight gain is typically associated with increases in arterial pressure [18].

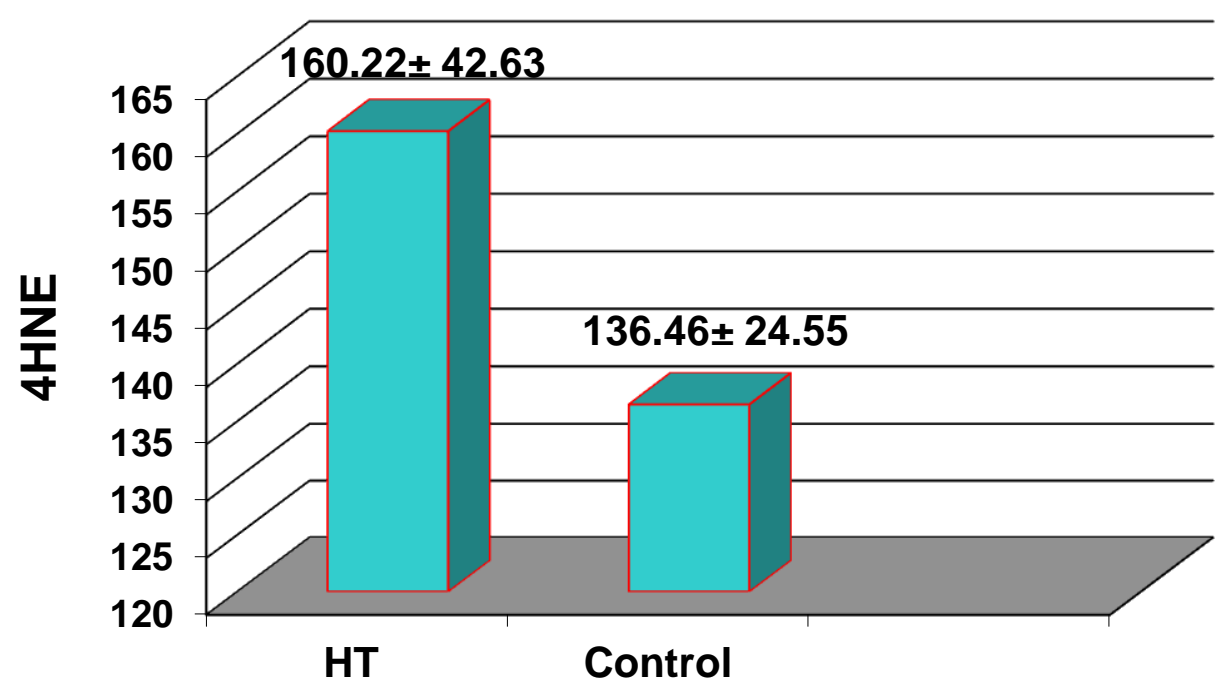

Fig.(1): Mean concentration (ng/L) Of oxidative marker $4 H \mathrm{NE}$ for studied groups.

The highly significant difference $(\mathrm{p}<0.01)$ of $4 \mathrm{HNE}$ in HT patient maybe due to high oxidative stress which generally causes damage to the membrane polyunsaturated fatty acids leading to the generation of $4 \mathrm{HNE}$. This result agrees with study (Juliane Cruz Campos et al 2015) [12]. 


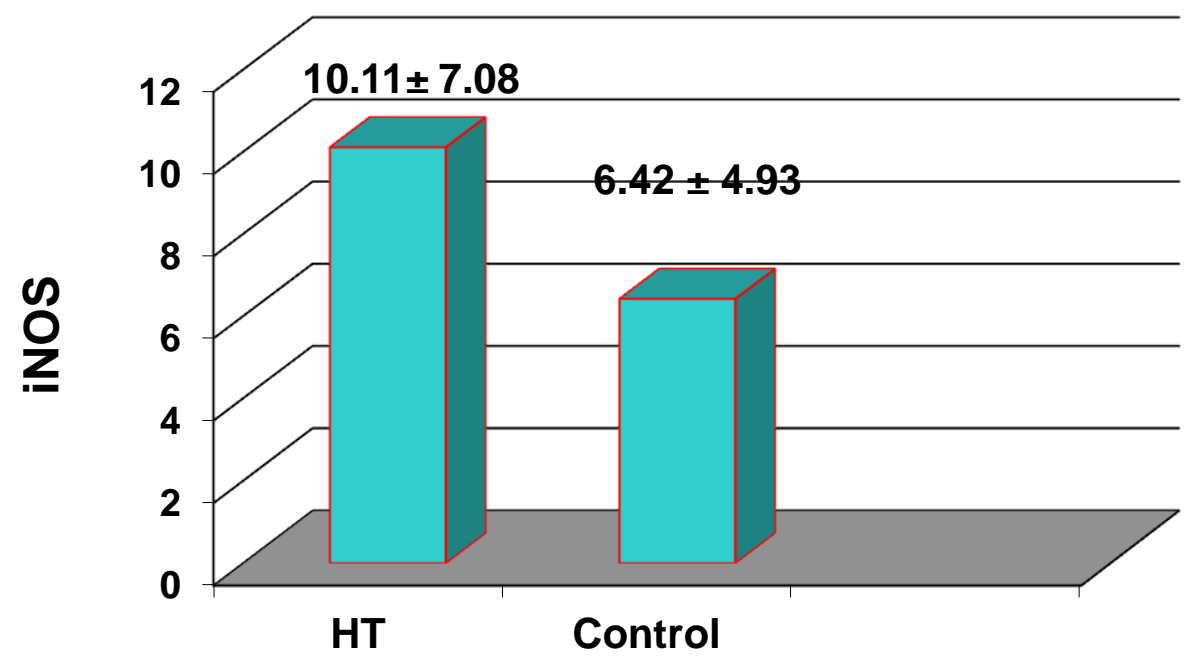

Fig.(2) Mean concentration (IU/ml) of INOS in the studied groups.

Our results showed a significant difference between hypertension patient and control groups $(\mathrm{p}<0.01)$. This result was agreed with (Caroline J. Smith et al) [19] who found a significant difference in iNOS levels in hypertensive patients.

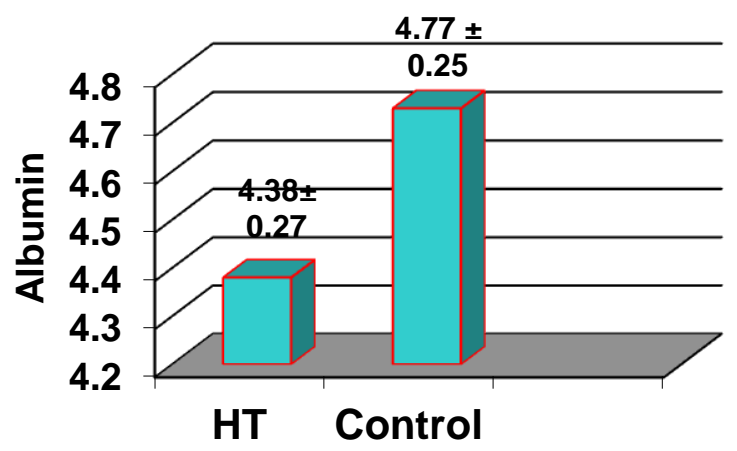

Fig. (3): Mean concentration ( $g / L)$ of Albumine in the studied groups.

Albumin contains a free sulfhydryl group, this forms a disulfide with several compounds like cysteine, homocysteine, or glutathione, Albumin is able to scavenge hydroxyl radicals [15], the decrease in albumin in patient is agreed with results of (Oda E. et al 2012) in Hypertension [15] who suggested that result is may be due to its function as antioxidant activity so the non-oxidized albumin is decrease in addition to negative acute phase protein, so inflammation is considered the principle cause of a decrease in the serum albumin [20]. The weak negative correlation between 4HNE and albumin in hypertension patient and control group agreed with (Giancarlo Aldini.et al 2006) who found a negative correlation between $4 \mathrm{HNE}$ and Albumin [21]. There is an evidence for a significant antioxidant activity of the represent the major and predominant circulating antioxidant in plasma known to be exposed to continuous oxidative stress.

There is also negative correlation between iNOS and albumin in patient and control group this result shown (Michael Poteser.et al 2004) who found a negative correlation between 4HNE and Albumin [22].

\section{Conclusion}

Based on this study is important nappy on ideal weight, because obesity considered main factors for heart disease and hardening of the arteries In addition, the effect of oxidative stress, which leads to high blood pressure.

\section{Acknowledgements}

The authors would like to thank Al-Nahrain University and Al-Yarmouk teaching hospital for the financial supports and research facilities.

\section{References}

[1] Caroline M., Introduction to Epidemiology: Distribution and Determinants of Disease. Cengage Learning, 6, 302-303. 2013.

[2] Ahmad A., Singhal U., Mohd M., Islam N.and Rivisi I., The Role of the Endogenous Antioxidant Enzymes and Malondialdehyde in Essential Hypertension. Journal of Clinical and Diagnostic Research, 7, 987990. 2013. 
[3] Yasunobu H. and Hiroshi S., Hypertension and Oxidative Stress. the Journal of the Japan Medical Association, 124, 15751579, 2000.

[4] Dhananjay V. Bhale, Manjusha D Hivre, Roshan K., Aasiya S., Devendra M. and Ashlesha B. Study of Oxidative Stress in Patients with Hypertension. International J. of Recent Trends in Science and Technology, 9, 157-158. 2013.

[5] Shiv K., Free Radicals and Antioxidants Human and Food System. Advances in Applied Science Research, 2, 129-135. 2011.

[6] José A., Oxidative Stress and Chronic Degenerative Disease a Role for Antioxidants. Janeza Trdine, 9, 34-35,2013.

[7] Ramalingam M., and Kim SJ., Reactive oxygen/ nitrogen species and their functional correlations in neurodegenerative diseases. Journal of Neural Transmission, 119 (8), 891-910. 2012.

[8] Chandra Kala., Syed Salman Ali., Abid Mohd., Sweety Rajpoot., Najam Ali Khan., Protection Against FCA Induced Oxidative Stress Induced DNA Damage as a Model of Arthritis and In vitro Anti-arthritic Potential of Costus speciosus Rhizome Extract. International Journal of Pharmacognosy and Phytochemical Research, 7 (2), 383389. 2015.

[9] Chandra Kala, Syed Salman Ali, Abid Mohd, Sweety Rajpoot, and Najam Ali Khan., Protection Against FCA Induced Oxidative Stress Induced DNA Damage as a Model of Arthritis and In vitro Antiarthritic Potential of Costus speciosus Rhizome Extract. International Journal of Pharmacognosy and Phytochemical Research, 7 (2), 383-389.2015.

[10] Kenneth M. Humphries and Luke I. Szweda., Selective Inactivation of $\alpha$ Ketoglutarate Dehydrogenase and Pyruvate Dehydrogenase: Reaction of Lipoic Acid with 4-Hydroxy-2-nonenal., American Chemical Society., 37 (4), 15835-15841. 1998.

[11] Sousa, T., Oliveira, S., Afonso, J., Morato, M., Patinha, D., Fraga, S., Carvalho, F. and Albino Teixeira A., Role of $\mathrm{H}(2) \mathrm{O}(2)$ in Hypertension. ReninAngiotensin System Activation and Renal
Medullary Disfunction Caused by Angiotensin II. Br J Pharmacol, 166(8), 2386-401. 2012.

[12] Juliane Cruz Campos., Tiago Fernandes., Luiz Roberto Grassmann Bechara., Nathalie Alves da Paixão., Patricia Chakur Brum., Edilamar Menezes de Oliveira., and Julio Cesar Batista Ferreira1., Increased Clearance of Reactive Aldehydes and Damaged Proteins in Hypertension-Induced Compensated Cardiac Hypertrophy. Institute of Biomedical Sciences, University of Sao Paulo, SP, Brazil (2015).

[13] W. Elremaly., I. Mohamed., T. Rouleau and J.C. Lavoie., Adding glutathione to parenteral nutrition prevents alveolar loss in newborn guinea pig. Free Radic. Biol. Med, 87, 274-281.2015.

[14] Taverna M., Anne-Lise M., Jean-Paul M. and Bertrand G., Specific antioxidant properties of human serum albumin, a Review. Annals of Intensive Care, 3, 1-7.2013.

[15] Oda E., Decreased serum albumin predicts hypertension in a Japanese health screening population. intern Med, 53(7), 655-660. 2012.

[16] Ahmad A., Singhal U., Mohd M., Islam N., and Rivisi I., The Role of the Endogenous Antioxidant Enzymes and Malondialdehyde in Essential Hypertension. J Clinical Diagnostic Research, 7, 987-990. 2013.

[17] Shuger S., Xuemei S., Timothy S., Rebecca A., and Meriwether S., Body Mass Index as a Predictor of Hypertension Incidence Among Initially Healthy Normotensive Women. American $\mathrm{J}$ of Hypertension, 21(6), 613-619.2008.

[18] Lavie C.J., Milani R.V., and Ventura H.O., Obesity and Cardiovascular Disease Risk Factor, Paradox, and Impact of Weight Loss. J American College of Cardiology,vol 53 no. 21.2009.

[19] Caroline J. Smith., Lakshmi Santhanam., Rebecca S. Bruning., Anna Stanhewicz., Dan E. Berkowitz., and Lacy A. Holowatz., Upregulation of Inducible Nitric Oxide Synthase Contributes to Attenuated Cutaneous Vasodilation in Essential Hypertensive Humans, 58,935-942. 2011. 
[20] Marjolaine R., Philippe R., Nihar R., Evelyne T., and Emmanuel B., The antioxidant properties of serum albumin. Review. FEBS Letters, 582, 1783-1787. 2008.

[21] Giancarlo Aldini., Luca Gamberoni., Marica Orioli., Giangiacomo Beretta., and Luca Regazzoni., Mass spectrometric characterization of covalent modification of human serum albumin by 4-hydroxy-trans2-nonenal. J. Mass Spectrom, 41, 11491161. 2006.

[22] Michael Poteser., and Ichiro Wakabayashi., Serum albumin induces iNOS expression and NO production in RAW 267.4 macrophages. British Journal of Pharmacology, 143, 143-151. 2004. 